\title{
Hybrid interval AHP-entropy method for electricity user evaluation in smart electricity utilization
}

\author{
Shouxiang WANG ${ }^{1}$, Leijiao GE ${ }^{1}$, Shengxia $\mathrm{CAI}^{2}$, Lei $\mathrm{WU}^{3}$
}

Abstract Smart electricity utilization (SEU) is one of the most important components in a smart grid. It is crucial to evaluate efficiency, safety, and demand response capability of electricity users to achieve the smart use of electricity. The analytic hierarchy process (AHP) uses subjective criteria to determine index weights in multi-criteria decisionmaking problems, while the entropy method provides objectivity in determining index weights. Taking into account the uncertainty of expert scoring and user data, a hybrid interval analytic hierarchy process (IAHP) and interval entropy (IE) method is proposed for electricity user evaluation (EUE). Specifically, in the proposed method, electricity users are evaluated in terms of energy efficiency, safety monitoring, and demand response. The weights of EUE indices are calculated under uncertainty. The proposed approach derives subjective weights of EUE indices

CrossCheck date: 7 September 2017

Received: 28 October 2016/ Accepted: 8 September 2017/Published online: 27 December 2017

(C) The Author(s) 2017. This article is an open access publication

$\triangle$ Leijiao GE

legendglj99@tju.edu.cn

Shouxiang WANG

sxwang@tju.edu.cn

Shengxia CAI

shengxia77@nankai.edu.cn

Lei WU

lwu@clarkson.edu

1 Key Laboratory of Smart Grid of Ministry of Education, Tianjin University, Tianjin 300072, China

2 Zhou Enlai School of Government, Nankai University, Tianjin 300071, China

3 Electrical and Computer Engineering Department, Clarkson University, Potsdam, NY 13699, USA by the IAHP with expert scoring as input data, and determines objective weights of EUE indices by the IE method with user data as inputs. In order to obtain the optimal combined index weights, the two weights are normalized by a selected weight factor. Numerical case studies illustrate the effectiveness and advantages of the proposed approach, which combines subjective and objective information to derive the optimal combined index weights.

Keywords Demand response, Interval analytic hierarchy process, Interval entropy method, Electricity user evaluation

\section{Introduction}

A smart grid comprises smart transmission, smart distribution and smart electricity utilization (SEU). The implementation of SEU is one of the most important features in a smart grid. Through building an interactive twoway communication system between the power supply company and electricity users, SEU provides a customerutility interface to realize intelligent homes and appliances linked to the grid. Customer participation in power markets can then be achieved using demand side management and distributed generation, which can optimize service quality, meet the diverse needs of customers, and improve power efficiency. With the acquisition of energy consumption information at the appliance level and two-way communication between users and the grid, demand response can be effectively applied in SEU.

Electric vehicles (EVs) and distributed generation are increasingly integrated into the electricity customer side, which increases diversity and uncertainty of electricity demand. Evaluating energy efficiency, safety and demand 
response capacity of electricity users becomes correspondingly more essential and complicated. The major issue for SEU is how to enhance energy efficiency and demand response capability of electricity users while ensuring electricity safety. Thus, proper evaluation indices for electricity users are crucial in SEU. Much research on evaluation indices has been reported, focused on the performance of either single pieces of electrical equipment [1] or the entire system [2, 3]. In [4], demand response strategies applied to air-conditioning peak load in Australia were described. Reference [5] evaluated the impact of electrical substations on the static and dynamic performance of electric power systems, while considering their possible operating states. Economic and low-carbon dayahead Pareto-optimal scheduling was discussed in [6] for wind farms integrated into power systems with the use of demand response. The future evolution of automated demand response systems in smart grids for a low-carbon economy was presented in [7]. However, existing literature rarely evaluates electricity users according to their distinct features such as load aggregation, regularity, and deferability, and pay very limited attention to the changing characteristics of electricity users in various scenarios. In addition, existing research largely ignores the interaction between electricity users and distributed generation and other new energy equipment when evaluating the demand response capability of electricity users.

The approach to calculating weights plays an important role in an evaluation index system, and can be categorized into subjective and objective methods. Much work has been done in the field of evaluation methods. In [8-14], the analytic hierarchy process (AHP) method [8], fuzzy AHP method [9-12], and entropy method [13, 14] were studied. Interval arithmetic (IA) is an efficient tool for handling "unknown but bounded" uncertainties. In [15], an automatic contingency selection approach was proposed, based on DC power flow calculations using affine arithmetic and considering load and generation uncertainties. Interval algorithms including interval matrices [16-18], interval models [19], and interval optimization algorithms [20] have presented, and applied in various power system applications [21-26]. In [27], the interval entropy (IE) method was applied in financial engineering analysis to assess the predictability of finance markets; it has potential for power system applications.

This paper focuses on electricity user evaluation (EUE) indices and weight calculation methods for them. The main contribution of this paper is twofold, as follows:

1) A comprehensive EUE index is presented for evaluating energy efficiency, safety monitoring, and demand response capability of electricity users in SEU.
2) A novel integrated interval analytic hierarchy process (IAHP) and IE method is proposed, which is suitable for deriving optimal subjective and objective weights while considering uncertainties in expert scoring and user data.

The rest of the paper is organized as follows. In Section 2, EUE indices are presented for energy efficiency, security monitoring, and demand response. Section 3 describes the hybrid IAHP and IE method to obtain optimal weights for EUE indices. Several case studies are provided in Section 4 for evaluating industrial users via the proposed method. Finally, conclusions are provided in Section 5.

\section{EUE indices}

Electricity users can be divided into four types: residential, small commercial, medium commercial, and large commercial and industrial users. Three categories of EUE indices are proposed to reflect their performance in terms of energy efficiency, safety monitoring, and demand response. The EUE indices include 3 first-level indices (i.e., $A_{1}, B_{1}$, and $C_{1}$ ), 15 second-level indices (i.e., $A_{11}-A_{21}$, $B_{11}-B_{12}$, and $C_{11}-C_{12}$ ), and 19 third-level indices (i.e., $B_{111}-B_{112}, B_{121}-B_{127}, C_{111}-C_{113}$, and $\left.C_{121}-C_{127}\right)$. The firstlevel EUE indices are presented in Fig. 1.

\subsection{Energy efficiency indices $A_{1}$}

Energy efficiency indices are formulated with several aims, as follows:

1) Presenting the energy situations of enterprises and users comprehensively.

2) Reflecting energy consumption issues and energy saving potentials.

3) Providing support for formulating energy saving programs.

4) Providing an accurate and scientific basis for energy saving transformation of the entire society.

The specific second-level energy efficiency indices are presented in Fig. 2 and described in detail as follows.

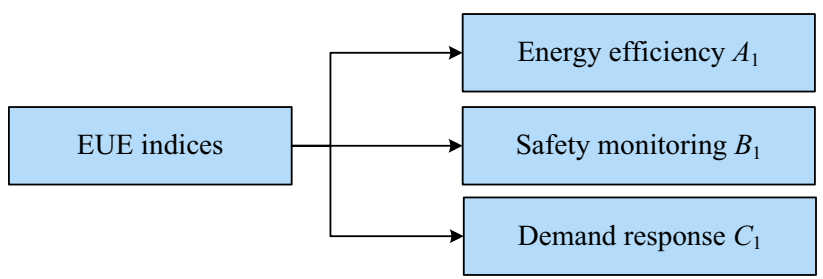

Fig. 1 First-level EUE indices 


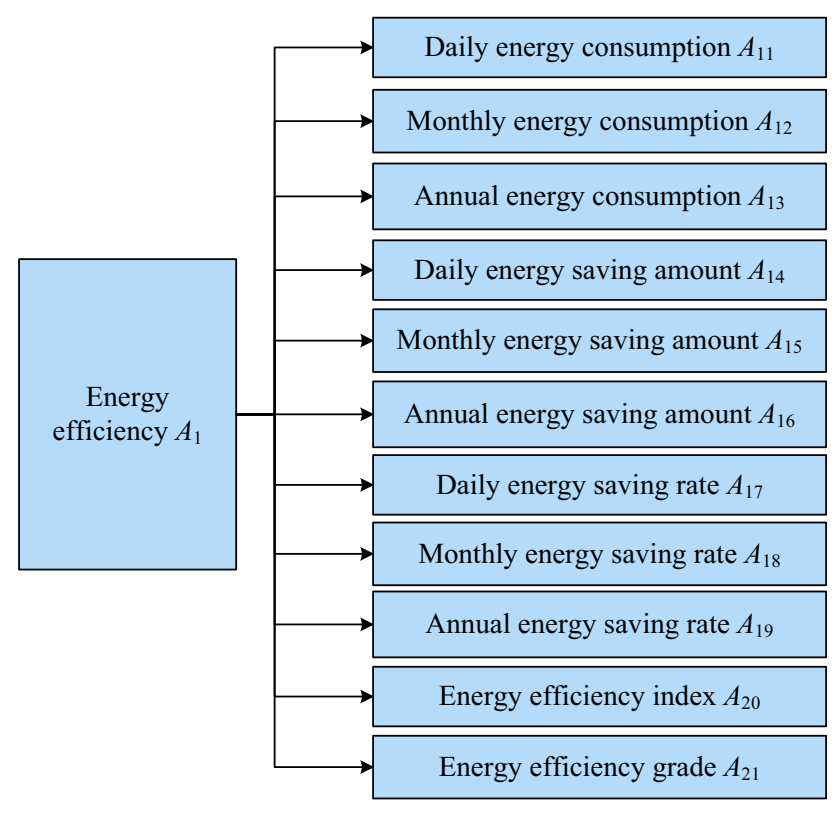

Fig. 2 Energy efficiency indices

The baseline energy consumption of equipment is usually quantified in terms of the actual energy consumption in each day $\left(A_{11}\right)$, each month $\left(A_{12}\right)$, or each year $\left(A_{13}\right)$, which can be directly measured by smart meters. The daily energy consumption $A_{11}$ is the total energy consumption of the user in each day, and is given by:

$A_{11}=\sum_{i=1}^{N} P_{i} h_{i}$

where $P_{i}$ and $h_{i}$ are the actual power and the working time period of the $i^{\text {th }}$ item of equipment in a day, respectively, and $N$ is the number of items of equipment. The monthly energy consumption $A_{12}$ and the annual energy consumption $A_{13}$ are the sum of daily energy consumptions in a month and in a year, respectively.

The amount of energy saving is the difference between the reference energy consumption and the actual energy consumption of an item of electrical equipment, which can also be measured for each day $\left(A_{14}\right)$, each month $\left(A_{15}\right)$, and each year $\left(A_{16}\right)$. Daily energy saving $A_{14}$ can be calculated by:

$A_{14}=\sum_{i=1}^{N} P_{i} h_{i}-\sum_{j=1}^{N} P_{j}^{\prime} h_{j}^{\prime}$

where $P_{j}^{\prime}$ and $h_{j}^{\prime}$ are the rated power and the planned working time period of the $j^{\text {th }}$ item of equipment, respectively. Similarly, the monthly energy saving $A_{15}$ is the difference between the reference and the actual energy consumptions in a month, and the annual energy saving $A_{16}$ is the difference between the reference and the annual actual energy consumptions in a year.
Energy saving rate reflects the energy saving level of equipment, including daily $\left(A_{17}\right)$, monthly $\left(A_{18}\right)$, and annual $\left(A_{19}\right)$ energy saving rates. The daily energy saving rate $A_{17}$ is the daily energy saving rate $A_{11}$ divided by the daily reference energy consumption, which is calculated as:

$A_{17}=\frac{\sum_{i=1}^{N} P_{i} h_{i}-\sum_{j=1}^{N} P_{j}^{\prime} h_{j}^{\prime}}{\sum_{j=1}^{N} P_{j}^{\prime} h_{j}^{\prime}} \times 100 \%$

Similarly, we can obtain the monthly energy saving rate $A_{18}$ and the annual energy saving rate $A_{19}$.

The energy efficiency index $A_{20}$ is defined as the daily actual energy consumption $A_{11}$ divided by the daily reference energy consumption, and is formulated as:

$A_{20}=\frac{\sum_{i=1}^{N} P_{i} h_{i}}{\sum_{j=1}^{N} P_{j}^{\prime} h_{j}^{\prime}} \times 100 \%$

Finally, the energy efficiency grade $A_{21}$ is a quantitative index for describing the significance of energy efficiency index values, and is defined as:

$A_{21}=\left\{\begin{array}{rr}1 & 0<A_{20} \leq 35 \% \\ 2 & 35 \%<A_{20} \leq 45 \% \\ 3 & 45 \%<A_{20} \leq 55 \% \\ 4 & 55 \%<A_{20} \leq 65 \% \\ 5 & 65 \%<A_{20} \leq 80 \%\end{array}\right.$

\subsection{Safety monitoring indices $B_{1}$}

Safety monitoring indices include equipment quality $B_{11}$ and equipment operating condition $B_{12}$, which are used to evaluate the safety level of equipment operation, investigate potential safety issues, and predict possible accidents. The specific second-layer indices are presented in Fig. 3.

Equipment quality $B_{11}$ includes the maintenance rate $B_{111}$ and quality grade $B_{112}$ for the entire system. Here, $B_{111}$ has the form as:

$B_{111}=\frac{\sum_{i=1}^{M} T_{i}}{\sum_{j=1}^{N} T_{j}^{\prime}} \times 100 \%$

where $M$ is the number of items of equipment under maintenance; $T_{i}$ is the maintenance time period of the $i^{\text {th }}$ item of equipment; $T_{j}^{\prime}$ is the operating time period of the $j^{\text {th }}$ item of equipment. Based on $B_{111}$ defined in (6), the equipment quality grade index $B_{112}$ defines four quality levels. When the value of $B_{111}$ is greater than $90 \%$, the 


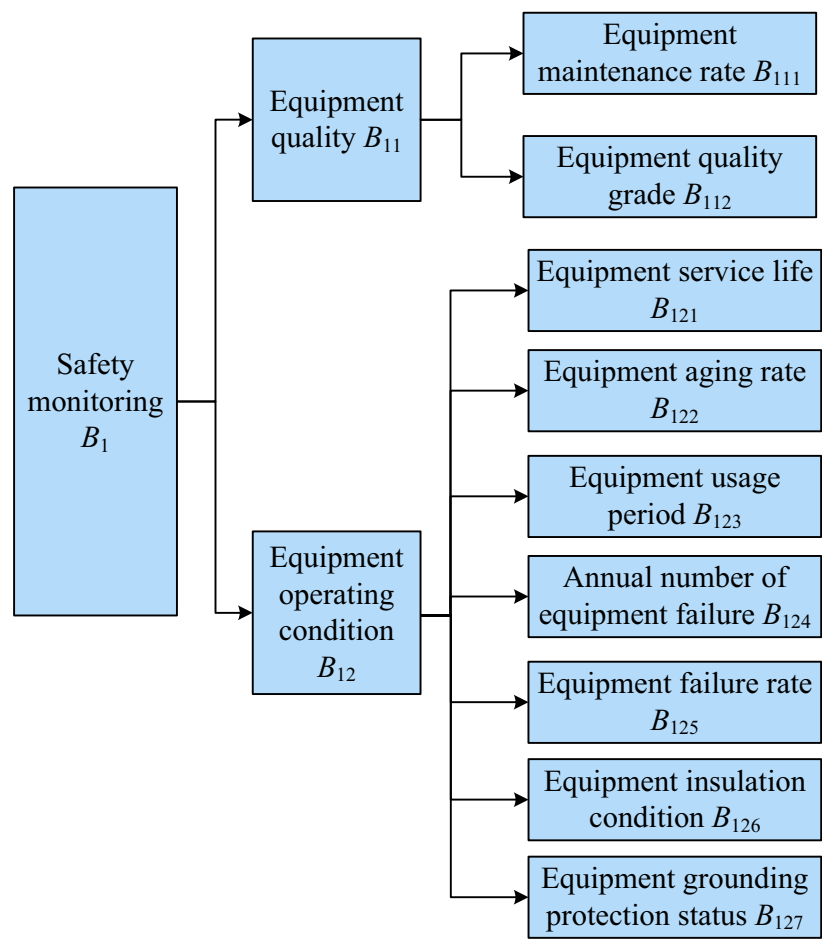

Fig. 3 Safety monitoring indices

state of $B_{112}$ is excellent. When the value of $B_{111}$ is greater than $70 \%$ and less than or equal $90 \%$, the state of $B_{112}$ is good. When the value of $B_{111}$ is greater than $60 \%$ and less than or equal $70 \%$, the state of $B_{112}$ is average. When the value of $B_{111}$ is less than or equal $60 \%$, the state of $B_{112}$ is poor.

The equipment operating condition index $B_{12}$ is the record of operation status, including service lifetime $B_{121}$, aging rate $B_{122}$, usage period $B_{123}$, annual number of failures $B_{124}$, failure rate $B_{125}$, insulation condition $B_{126}$, and grounding protection status $B_{127}$. Indices $B_{121}, B_{124}, B_{125}$, $B_{126}$, and $B_{127}$ can be directly recorded by operation and maintenance personnel, while $B_{122}$ can be calculated via (7), as follows. $B_{122}$ is defined by:

$B_{122}=\frac{Y_{1}}{Y_{2}} \times 100 \%$

where $Y_{1}$ is the actual life time of the equipment and $Y_{2}$ represents the standard service life time of the equipment. According to $B_{122}$, index $B_{123}$ describes three periods of equipment usage, including new, maturity, and decline. When the value of $B_{122}$ is less than or equal 2, the state of $B_{123}$ is new. When the value of $B_{122}$ is greater than 2 and less than or equal 5 , the state of $B_{123}$ is maturity. When the value of $B_{122}$ is greater than 5 and less than or equal 10 , the state of $B_{123}$ is decline.

\subsection{Demand response indices $C_{1}$}

Demand response represents the most useful kind of demand side management. Demand response indices $C_{1}$ include the desire index $C_{11}$ and the ability index $C_{12}$, as shown in Fig. 4.

The demand response desire index $C_{11}$ includes annual allowable outage time $C_{111}$, annual allowable outage number $C_{112}$, and annual allowable outage duration $C_{113}$.

The demand response ability index $C_{12}$ includes controllable load capacity $C_{121}$, controllable load proportion $C_{122}$, controllable power supply capacity $C_{123}$, controllable energy storage capacity $C_{124}$, energy storage proportion $C_{125}$, peak clipping capacity $C_{126}$, and valley filling capacity $C_{127}$. Indices $C_{121}, C_{123}, C_{124}, C_{126}$, and $C_{127}$ can be directly recorded from user-side smart meters or user design documents, while $C_{122}$ and $C_{125}$ can be calculated via (8) and (9), as follows. $C_{122}$ is defined by:

$C_{122}=\frac{C_{121}}{C_{\text {total }}} \times 100 \%$

where $C_{121}$ is the controllable load capacity, given by the rated capacity of controllable load; $C_{\text {total }}$ is the total load capacity. $C_{125}$ is formulated as:

$C_{125}=\frac{C_{\text {energy }}}{C_{\text {total }}} \times 100 \%$

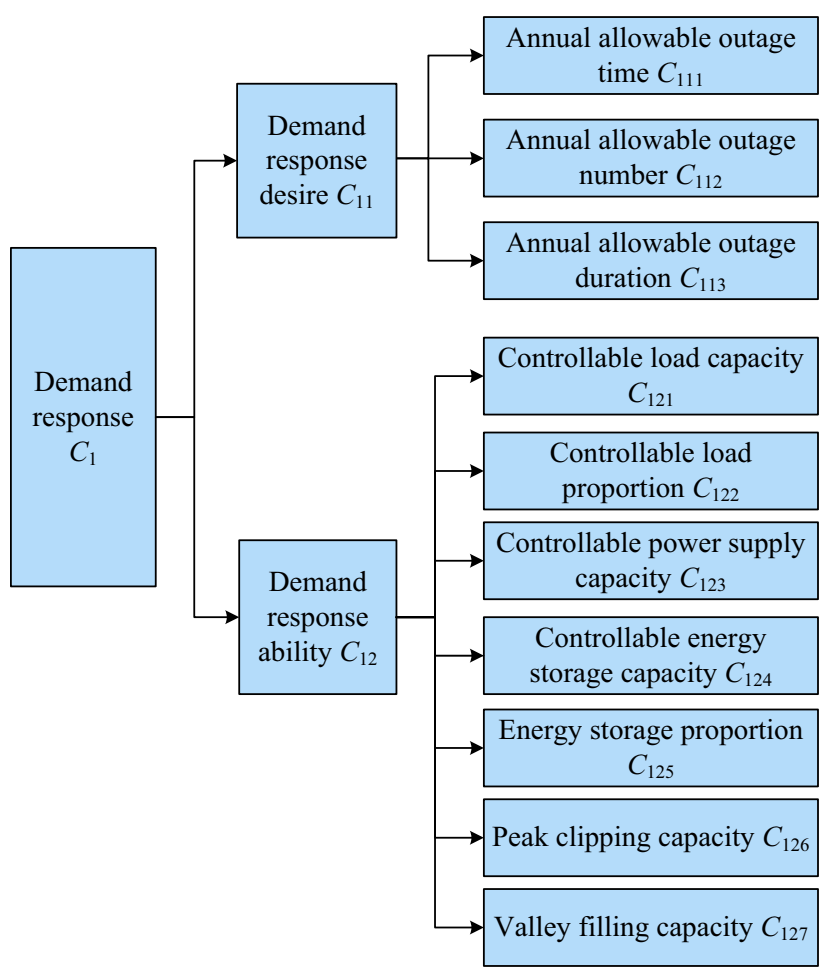

Fig. 4 Demand response indices 
where $C_{\text {energy }}$ is the energy storage capacity.

\subsection{Choice of indices}

The EUE indices have theoretically covered the quantitative evaluation on all electricity users with regard to energy efficiency, safety monitoring and demand response. However, some of the second-level or third-level indices may be difficult to obtain for some specific electricity users, and some may be less necessary for practical evaluation. Hence the choice of proper EUE indices is important. The following steps will help to determine the indices selected:

1) The calculation of EUE indices is carried out according to the feasibility of data acquisition of actual electricity users.

2) After accumulating enough evaluation data for EUE, a frequency analysis method is used to determine the final EUE index group that is suitable for different user categories. This method is a conventional mathematical method, which is not tired.

\section{A hybrid interval AHP-entropy method for EUE}

In this section, we focus on the important problem of determining the weights of indices, in order to achieve effective EUE. Existing methods for solving the weight problem can be classified into two main categories: subjective and objective methods. A subjective method such as the AHP approach can incorporate the subjective view of users, but potentially with random and large arbitrary variations. In contrast, an objective method such as the entropy approach can use physical data and determine weight values from them. However, it may be difficult to incorporate suggestions based on objective criteria. Moreover, both AHP and entropy methods are analytical approaches designed to handle deterministic situations, and they may not be capable of dealing with uncertainties in the index calculation procedure. In order to calculate weights of evaluation indices, this paper proposes a new method that combines IAHP and IE, which can balance subjective and objective points of view and properly handle uncertainties. The detailed procedure includes three main parts: IAHP, IE, and comprehensive interval weight, which are presented below.

\subsection{IAHP method}

The IAHP method comprises the following procedures.
Step 1: Interval judgment matrix $[\boldsymbol{A}]$ derivation.

According to the EUE index system, a three-layer index system is first established, including the target layer, the criteria layer, and the scheme layer. Next, $N$ experts are invited to score the indices by comparing each pair with the predefined index hierarchies. Finally, according to the pairwise comparison of the importance of individual indices in the selected layer to indices in the upper layer, the judgment matrix $[\boldsymbol{A}]$ is established. The interval judgment matrix $[A]$ of size $n \times n$ has the form as:

$[\boldsymbol{A}]=\left[\begin{array}{cccc}{\left[a_{11}^{\prime}\right]} & {\left[a_{12}^{\prime}\right]} & \cdots & {\left[a_{1 n}^{\prime}\right]} \\ {\left[a_{21}^{\prime}\right]} & {\left[a_{22}^{\prime}\right]} & \cdots & {\left[a_{2 n}^{\prime}\right]} \\ \vdots & \vdots & & \vdots \\ {\left[a_{n 1}^{\prime}\right]} & {\left[a_{n 2}^{\prime}\right]} & \cdots & {\left[a_{n n}^{\prime}\right]}\end{array}\right]$

where $n$ denotes the total number of indices in the selected index layer. The value of each element $\left[a_{i j}^{\prime}\right]$ in $[\boldsymbol{A}]$ is determined by:

$\left[a_{i j}^{\prime}\right]=\left[a_{i j}, \mu\right] \quad 0<\mu<1$

where $i=1,2, \ldots, N$ and $j=1,2, \ldots, N$. In general, an interval number $\left[a_{i j}^{\prime}\right]$ is represented by the midpoint $a_{i j}$ and the width $\mu$. $\mu$ will be provided by experts according to the degree of uncertainty and vagueness.

Step 2: Interval proportional scale derivation.

The pairwise comparison of expert scoring is represented using the interval proportional scale shown in Table 1.

When the $i^{\text {th }}$ index is regarded to be more important than the $j^{\text {th }}$ one in the selected index layer, which results in $\left[a_{i j}^{\prime}\right] \geq 1, i \neq j$, the corresponding element of $[\boldsymbol{A}]$ can be determined by:

$\left\{\begin{array}{l}{\left[a_{i j}^{\prime}\right]=\left[a_{i j}^{\prime \mathrm{L}}, a_{i j}^{\prime} \mathrm{U}\right]} \\ a_{i j}^{\mathrm{L}}=a_{i j}-\mu \\ a_{i j}^{\prime} \mathrm{U}=a_{i j}+\mu\end{array}\right.$

Conversely, if the $j^{\text {th }}$ index is thought to be more important than the $i^{\text {th }}$ one, which results in $1 /\left[a_{i j}^{\prime}\right]$ $\geq 1, j \neq i$, the corresponding element of $[A]$ can be calculated as:

$\left\{\begin{array}{l}{\left[a_{i j}^{\prime}\right]=\left[a_{i j}^{\prime} \mathrm{L}, a_{i j}^{\prime} \mathrm{U}\right]} \\ a_{i j}^{\prime} \mathrm{L}=\frac{1}{a_{i j}+\mu} \\ a_{i j}^{\prime} \mathrm{U}=\frac{1}{a_{i j}-\mu}\end{array}\right.$

Step 3: Eigenvector derivation.

The power method is used to calculate the largest eigenvalue $\left[\lambda_{\max }\right]$ and the corresponding eigenvector $[\xi]$ of the judgment matrix $[\boldsymbol{A}]$, which satisfy: 
Table 1 Interval proportional scale

\begin{tabular}{lcc}
\hline Importance of index $i$ compared to index $j$ & Midpoint of interval $a_{i j}$ & Interval width $\mu$ \\
\hline Equal importance & 1 & $0<\mu<1$ \\
Slightly higher importance & 3 & $0<\mu<1$ \\
Higher importance & 5 & $0<\mu<1$ \\
Much higher importance & 7 & $0<\mu<1$ \\
Absolute importance & 9 & $0<\mu<1$ \\
Midpoint of adjacent judgment & $2,4,6,8$ & $0<\mu<1$ \\
\hline
\end{tabular}

$\boldsymbol{A} \xi=\lambda_{\max } \xi$

Step 4: Consistency check (CR).

We use $C R$ to denote the value of the relative consistency test, which is calculated as:

$C R=\frac{C I}{R I}$

where $C I=\left(\lambda_{\max }-n\right) /(n-1)$ and the freedom index $R I$ takes values according to Table 2 . Generally, a smaller $C R$ indicates a better consistency of $[\boldsymbol{A}]$. If $C R$ of $\boldsymbol{A}$ is less than $0.1,[\boldsymbol{A}]$ is feasible and consequently passes the consistency test. Otherwise, $[\boldsymbol{A}]$ fails to pass the test, and the procedure goes back to Step 2 for reconstructing the qualified judgment matrix $[\boldsymbol{A}]$.

Step 5: Interval weight of each index $\left[w_{\text {IAHP }}\right]$

With $N$ experts, by repeating the above procedures, we can obtain $N$ eigenvectors $\left[w_{i}\right], i=1,2, \ldots, N$. Then, we calculate the average value of these eigenvectors as:

$\left[w_{\text {IAHP }}\right]=\frac{1}{N} \sum_{i=1}^{N}\left[w_{i}\right]$

This can be used as the interval weight of the selected index layer.

\subsection{IE method}

The IE method includes the following procedures.

Step 1: Decision-making matrix $[\boldsymbol{B}]$ derivation.

Initializing an index set $Q=\left\{Q_{1}, Q_{2}, \ldots, Q_{m}\right\}$ which includes the second and the third layer indices of the EUE index system, where $m$ is the total number of indices. By repeating $n$ times we obtain the object set $S_{i j}=\left\{S_{1 j}, S_{2 j}, \ldots\right.$,

Table 2 Freedom index

\begin{tabular}{llllllllll}
\hline$n$ & 1 & 2 & 3 & 4 & 5 & 6 & 7 & 8 & 9 \\
\hline$R I$ & 0 & 0 & 0.58 & 0.96 & 1.12 & 1.24 & 1.32 & 1.41 & 1.45 \\
\hline
\end{tabular}

$\left.S_{n j}\right\}$ for each index $Q_{j}$. The interval decision-making matrix $[\boldsymbol{B}]$ is formulated as:

$[\boldsymbol{B}]=\left[\begin{array}{cccc}{\left[b_{11}\right]} & {\left[b_{12}\right]} & \cdots & {\left[b_{1 n}\right]} \\ {\left[b_{21}\right]} & {\left[b_{22}\right]} & \cdots & {\left[b_{2 n}\right]} \\ \vdots & \vdots & & \vdots \\ {\left[b_{m 1}\right]} & {\left[b_{m 2}\right]} & \cdots & {\left[b_{m n}\right]}\end{array}\right]$

where $\left[b_{i j}\right]=\left[b_{i j}^{\mathrm{L}}, b_{i j}^{\mathrm{U}}\right]$ is the interval value of the $i^{\text {th }}$ evaluation for the $j^{\text {th }}$ index $Q_{j}$.

Step 2: Data normalization.

The interval decision-making matrix $[\boldsymbol{B}]$ is normalized by $[\boldsymbol{P}]$, which can be depicted as:

$[\boldsymbol{P}]=\left[\begin{array}{cccc}{\left[p_{11}\right]} & {\left[p_{12}\right]} & \cdots & {\left[p_{1 n}\right]} \\ {\left[p_{21}\right]} & {\left[p_{22}\right]} & \cdots & {\left[p_{2 n}\right]} \\ \vdots & \vdots & & \vdots \\ {\left[p_{m 1}\right]} & {\left[p_{m 2}\right]} & \cdots & {\left[p_{m n}\right]}\end{array}\right]$

An index $Q_{j}$ is called a profitability index if the profitability is increased with the increase of the index. On the other hand, an index $Q_{j}$ is called a cost index if the cost is decreased with the decrease of the index.

If index $Q_{j}$ is a profitability index, the corresponding row elements of $[\boldsymbol{P}]$ are given by:

$\left[p_{i j}\right]=\frac{\left[b_{i j}\right]}{\sum_{k=1}^{m}\left[b_{k j}\right]}$

Since $\left[p_{i j}\right]$ is an interval, its lower bound $p_{i j}^{\mathrm{L}}$ and upper bound $p_{i j}^{\mathrm{U}}$ can be expressed as:

$\left\{\begin{array}{l}p_{i j}^{\mathrm{L}}=\frac{b_{i j}^{\mathrm{L}}}{\sum_{k=1}^{m} b_{k j}^{\mathrm{U}}} \\ p_{i j}^{\mathrm{U}}=\frac{b_{i j}^{\mathrm{U}}}{\sum_{k=1}^{m} b_{k j}^{\mathrm{L}}}\end{array}\right.$

If the index $Q_{j}$ is a cost index, the corresponding row elements of $[\boldsymbol{P}]$ are given by: 
$\left[p_{i j}\right]=\frac{1}{\left[b_{i j}\right] \sum_{k=1}^{m} \frac{1}{\left[b_{i j}\right]}}$

Lower and upper bounds of $\left[p_{i j}\right]$ can be expressed as:

$\left\{\begin{array}{l}p_{i j}^{\mathrm{L}}=\frac{1}{b_{i j}^{\mathrm{U}} \sum_{k=1}^{m} \frac{1}{b_{k j}^{\mathrm{L}}}} \\ p_{i j}^{\mathrm{U}}=\frac{1}{b_{i j}^{\mathrm{L}} \sum_{k=1}^{m} \frac{1}{b_{k j}^{\mathrm{U}}}}\end{array}\right.$

Step 3: Index entropy derivation.

The entropy of the $j^{\text {th }}$ index $Q_{j}$ is calculated as:

$\left[H_{j}\right]=-k \sum_{i=1}^{m}\left[p_{i j}\right] \ln \left(\left[p_{i j}\right]\right)$

where $k=\ln (m)-1$ and $p_{i j} \ln \left(p_{i j}\right)=0$. In order to calculate the IE of the index $\left[H_{j}\right]=\left[H_{j}^{\mathrm{L}}, H_{j}^{\mathrm{U}}\right]$, two optimizations are performed:

$$
\begin{aligned}
& \left\{\begin{array}{l}
H_{j}^{\mathrm{L}}=\min \left\{-k \sum_{i=1}^{m}\left[p_{i j}\right] \ln \left(\left[p_{i j}\right]\right)\right\} \\
\text { s.t. } p_{i j}^{\mathrm{L}} \leq p_{i j} \leq p_{i j}^{\mathrm{U}} \quad i=1,2, \cdots, m \\
\sum_{i=1}^{m} p_{i j}=1
\end{array}\right. \\
& \left\{\begin{array}{l}
H_{j}^{\mathrm{U}}=\max \left\{-k \sum_{i=1}^{m}\left[p_{i j}\right] \ln \left(\left[p_{i j}\right]\right)\right\} \\
\text { s.t. } p_{i j}^{\mathrm{L}} \leq p_{i j} \leq p_{i j}^{\mathrm{U}} \quad i=1,2, \cdots, m \\
\sum_{i=1}^{m} p_{i j}=1
\end{array}\right.
\end{aligned}
$$

Step 4: Interval weight of entropy of index [ $\left.w_{\mathrm{IE}}\right]$ derivation.

After deriving the IE $\left[H_{j}\right]=\left[H_{j}^{\mathrm{L}}, H_{j}^{\mathrm{U}}\right]$, the IE $\left[w_{\mathrm{IE}}\right]=$ $\left[w_{j}\right]=\left[w_{j}^{\mathrm{L}}, w_{j}^{\mathrm{U}}\right]$ of the $j^{\text {th }}$ index $Q_{j}$ can be calculated as:

$\left[w_{j}\right]=\frac{1-\left[H_{j}\right]}{n-\sum_{j=1}^{n}\left[H_{j}\right]}$

It can also be expressed as:

$$
\left\{\begin{array}{l}
w_{j}^{\mathrm{L}}=\frac{1-H_{j}^{\mathrm{U}}}{n-\sum_{j=1}^{n} H_{j}^{\mathrm{L}}} \\
w_{j}^{\mathrm{U}}=\frac{1-H_{j}^{\mathrm{L}}}{n-\sum_{j=1}^{n} H_{j}^{\mathrm{U}}}
\end{array}\right.
$$

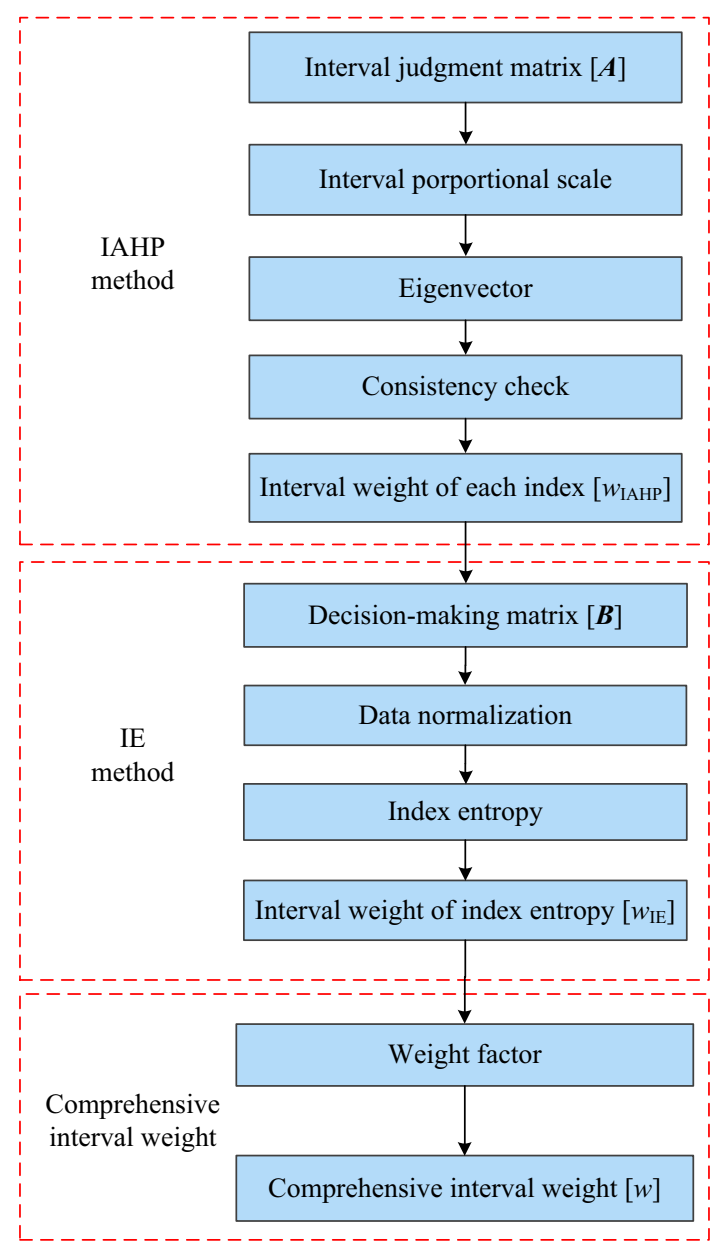

Fig. 5 Flowchart of comprehensive interval weight calculation

\subsection{Comprehensive interval weight}

In order to leverage advantages of IAHP for determining subjective weights and IE for setting objective weights, the weight factor $\theta$ is used to combine IAHP and IE methods. The interval weight $[w]$ for comprehensive index evaluation can be calculated as:

$\left\{\begin{array}{l}{[w]=\theta\left[w_{\mathrm{IAHP}}\right]+(1-\theta)\left[w_{\mathrm{IE}}\right]} \\ 0 \leq \theta \leq 1\end{array}\right.$

Table 3 Expert scoring for an industrial user

\begin{tabular}{llllll}
\hline Index & \multicolumn{4}{l}{ Expert scoring } \\
\cline { 2 - 6 } & $A_{11}$ & $A_{14}$ & $A_{17}$ & $A_{20}$ & $A_{21}$ \\
\hline$A_{11}$ & {$[1,0]$} & {$[2,0.6]$} & & & \\
$A_{14}$ & & {$[1,0]$} & & & \\
$A_{17}$ & {$[3,0.4]$} & {$[3,0.6]$} & {$[1,0]$} & & \\
$A_{20}$ & {$[4,0.3]$} & {$[4,0.8]$} & {$[5,0.2]$} & {$[1,0]$} & \\
$A_{21}$ & {$[6,0.5]$} & {$[7,0.1]$} & {$[8,0.7]$} & {$[8,0.9]$} & {$[1,0]$} \\
\hline
\end{tabular}


where $[w]$ denotes the comprehensive interval weight, which is a function of $\theta$. When $\theta=1$ or $\theta=0$, it reduces to the pure IAHP or IE method. The calculation procedure is shown in Fig. 5.

\section{Case study}

\subsection{Data sets}

In order to validate the proposed hybrid interval AHPentropy method, a typical industrial user is considered in numerical case studies. In consideration of the characteristics of industrial users, energy efficiency indices are mainly taken into account including $A_{11}, A_{14}, A_{17}, A_{20}$, and $A_{21}$. The expert scoring in Table 3 shows the pairwise comparison among the selected energy efficiency indices. The composition and operating condition of the industrial user's equipment is listed in Table 4 . The electricity consumption profile is shown in Fig. 6, which shows the maximum, average, and minimum electricity consumption of the industrial user during a week in May.

According to the expert scoring results and the characteristics of the data for this industrial user, judgment matrices of the AHP and IAHP are presented in Table 5, and normalized decision making matrices of the entropy and IE methods are presented in Table 6, calculated as described in Section 3. $S_{\text {Mon }}$ is the standardized actual value of the indices on Monday, the meaning of $S_{\text {Tue }}$ is the same, and so on.

\subsection{Comparison between AHP and the proposed hybrid method}

The weight of AHP method is calculated from the input data in Table 5. The weight of the proposed hybrid method is calculated from the input data in Table 5 and Table 6 , together with the weight factor of $\theta=0.8$. The results are listed in Table 7.

Several conclusions can be made from Table 7:

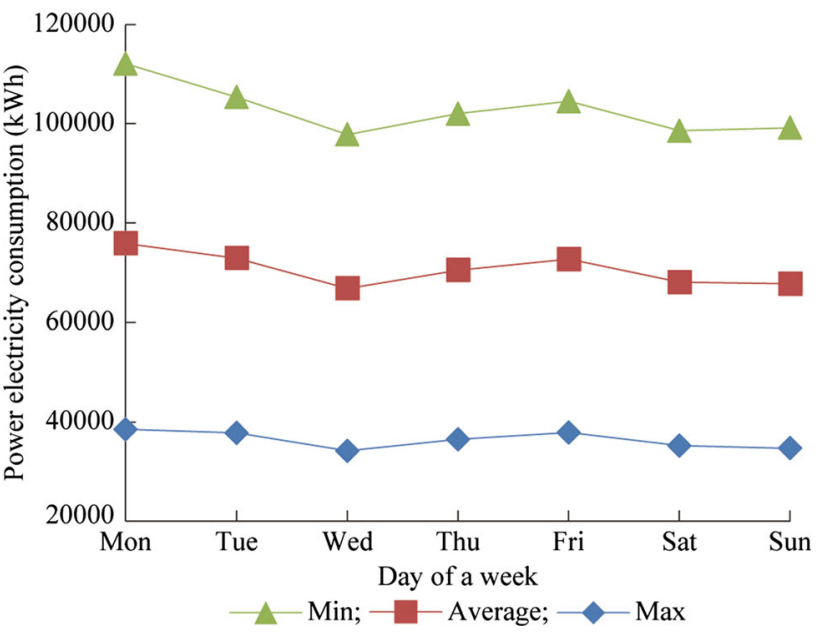

Fig. 6 Electricity consumption data of an industrial user for one week

1) The trends of weights in both AHP and the proposed hybrid method are consistent.

2) For each index, the weight obtained from AHP is within the weight interval from the proposed hybrid method. The hybrid method can exploit the fuzziness of the weights, and thus is useful for judging the expert scoring.

3) The assessment result for electricity utilization is an interval, which accords with common sense. For instance, a student is considered excellent when his scoring in a test is within the interval of $[90,100]$.

4) Results from the AHP rely strongly on the subjective experience of experts. On the other hand, the proposed hybrid method combines the actual data of the equipment and the expert scoring, and also considers the fuzziness of the experts and the equipment. Thus, it can effectively deal with the bias of experts, and in turn is more appropriate for the assessment.

Table 4 User equipment components

\begin{tabular}{llrlr}
\hline $\begin{array}{l}\text { Serial } \\
\text { number }\end{array}$ & Equipment name & $\begin{array}{l}\text { Total equipment capacity } \\
(\mathrm{kW})\end{array}$ & $\begin{array}{l}\text { Average daily working } \\
\text { hours }\end{array}$ & $\begin{array}{l}\text { Benchmark of daily power consumption or } \\
\text { generation }(\mathrm{kWh})\end{array}$ \\
\hline 1 & Power equipment & 3000 & {$[8,12]$} & 36000 \\
2 & Lighting & 20 & {$[10,15]$} & 300 \\
3 & Central air-conditioning & 380 & {$[8,15]$} & 5700 \\
4 & Distributed photovoltaic & 200 & {$[2,8]$} & 1600 \\
\hline
\end{tabular}


Table 5 Judgment matrices of AHP and IAHP methods

\begin{tabular}{|c|c|c|c|c|c|c|}
\hline Method & Index & $A_{11}$ & $A_{14}$ & $A_{17}$ & $A_{20}$ & $A_{21}$ \\
\hline \multirow[t]{5}{*}{ AHP } & $A_{11}$ & 1.000 & 2.000 & 0.333 & 0.250 & 0.167 \\
\hline & $A_{14}$ & 0.500 & 1.000 & 0.333 & 0.250 & 0.143 \\
\hline & $A_{17}$ & 3.000 & 3.000 & 1.000 & 0.200 & 0.125 \\
\hline & $A_{20}$ & 4.000 & 4.000 & 5.000 & 1.000 & 0.111 \\
\hline & $A_{21}$ & 6.000 & 7.000 & 8.000 & 9.000 & 1.000 \\
\hline \multirow[t]{5}{*}{ IAHP } & $A_{11}$ & {$[1.000,1.000]$} & {$[1.400,2.600]$} & {$[0.294,0.385]$} & {$[0.233,0.270]$} & {$[0.154,0.182]$} \\
\hline & $A_{14}$ & {$[0.385,0.714]$} & {$[1.000,1.000]$} & {$[0.278,0.417]$} & {$[0.208,0.313]$} & {$[0.141,0.145]$} \\
\hline & $A_{17}$ & {$[2.600,3.400]$} & {$[2.400,3.600]$} & {$[1.000,1.000]$} & {$[0.192,0.208]$} & {$[0.115,0.137]$} \\
\hline & $A_{20}$ & {$[3.700,4.300]$} & {$[3.200,4.800]$} & {$[4.800,5.200]$} & {$[1.000,1.000]$} & {$[0.112,0.141]$} \\
\hline & $A_{21}$ & {$[5.500,6.500]$} & {$[6.900,7.100]$} & {$[7.100,8.700]$} & {$[7.100,8.900]$} & {$[1.000,1.000]$} \\
\hline
\end{tabular}

Table 6 Normalized decision-making matrices of entropy and IE methods

\begin{tabular}{lllllll}
\hline Method & Value of indices & $A_{11}$ & $A_{14}$ & $A_{17}$ & $A_{20}$ & $A_{21}$ \\
\hline Entropy & $S_{\text {Mon }}$ & 0.1384 & 0.1044 & 0.1044 & 0.1473 & 0.1563 \\
& $S_{\text {Tue }}$ & 0.1367 & 0.0891 & 0.0891 & 0.1490 & 0.1563 \\
& $S_{\text {Wed }}$ & 0.1511 & 0.2125 & 0.2125 & 0.1439 & 0.1250 \\
& $S_{\text {Thu }}$ & 0.1416 & 0.1337 & 0.1337 & 0.1493 & 0.1563 \\
& $S_{\text {Fri }}$ & 0.1365 & 0.0871 & 0.0871 & 0.1388 & 0.1563 \\
& $S_{\text {Sat }}$ & 0.1468 & 0.1779 & 0.1779 & 0.1368 & 0.1250 \\
& $S_{\text {Sun }}$ & 0.1489 & 0.1954 & 0.1954 & {$[0.1421,0.1712]$} & {$[0.1290,0.2273]$} \\
& $S_{\text {Mon }}$ & {$[0.1188,0.1433]$} & {$[0.0328,0.1495]$} & {$[0.0328,0.1495]$} & {$[0.1272,0.1681]$} & {$[0.0968,0.2273]$} \\
& $S_{\text {Tue }}$ & {$[0.1210,0.1601]$} & {$[0.0448,0.2851]$} & {$[0.0448,0.2851]$} & {$[0.1217,0.1521]$} & {$[0.0968,0.1818]$} \\
& $S_{\text {Wed }}$ & {$[0.1338,0.1674]$} & {$[0.1069,0.3354]$} & {$[0.1069,0.3354]$} & {$[0.1236,0.1624]$} & {$[0.0968,0.1818]$} \\
& $S_{\text {Thu }}$ & {$[0.1254,0.1647]$} & {$[0.0673,0.3175]$} & {$[0.0673,0.3068]$} & {$[0.1250$} \\
& $S_{\text {Fri }}$ & {$[0.1209,0.1632]$} & {$[0.0438,0.3068]$} & {$[0.0438,0.3068]$} & {$[0.1248,0.1684]$} & {$[0.0968,0.2273]$} \\
& $S_{\text {Sat }}$ & {$[0.1299,0.1701]$} & {$[0.0895,0.3532]$} & {$[0.0895,0.3532]$} & {$[0.1197,0.1566]$} & {$[0.0968,0.1818]$} \\
& $S_{\text {Sun }}$ & {$[0.1319,0.1653]$} & {$[0.0983,0.3211]$} & {$[0.0983,0.3211]$} & {$[0.1232,0.1543]$} & {$[0.0968,0.1818]$} \\
\hline
\end{tabular}

Table 7 Weights of AHP and the proposed hybrid method

\begin{tabular}{lll}
\hline Index & $w_{\text {AHP }}$ & {$\left[w_{\text {hybrid }}\right]$} \\
\hline$A_{11}$ & 0.0709 & {$[0.0686,0.0735]$} \\
$A_{14}$ & 0.0388 & {$[0.0315,0.0519]$} \\
$A_{17}$ & 0.1590 & {$[0.1493,0.1686]$} \\
$A_{20}$ & 0.2561 & {$[0.2538,0.2607]$} \\
$A_{21}$ & 0.4752 & {$[0.4656,0.4764]$} \\
\hline
\end{tabular}

\subsection{Comparison among IAHP, IE, and proposed hybrid method}

Using the calculation procedure described in Section 3, the index weights from IAHP, IE, and the proposed hybrid method are presented in Table 8 . The weight factor $\theta$ is set as 0.5 .
Table 8 The weight of equipment energy efficiency index by IAHP, IE, and the proposed hybrid method

\begin{tabular}{llll}
\hline Index & {$\left[w_{\text {IAHP }}\right]$} & {$\left[w_{\text {IE }}\right]$} & {$\left[w_{\text {hybrid }}\right]$} \\
\hline$A_{11}$ & {$[0.0686,0.0735]$} & {$[0.1276,0.4034]$} & {$[0.0981,0.2385]$} \\
$A_{14}$ & {$[0.0315,0.0519]$} & {$[0.0325,0.5658]$} & {$[0.0320,0.3089]$} \\
$A_{17}$ & {$[0.1493,0.1686]$} & {$[0.0325,0.5651]$} & {$[0.0909,0.3669]$} \\
$A_{20}$ & {$[0.2538,0.2607]$} & {$[0.1278,0.4033]$} & {$[0.1908,0.3320]$} \\
$A_{21}$ & {$[0.4656,0.4764]$} & {$[0.0935,0.4786]$} & {$[0.2796,0.4775]$} \\
\hline
\end{tabular}

Several observations can be made from Table 8 .

1) The trends of weights in both IAHP and the proposed hybrid method are consistent. However, the trend of weights in IE method is different. The IE method is an objective approach, and the results solely rely on the 
observed data set. That is, different data sets for the same electricity user may lead to different weights.

2) In theory, the subjective methods of the IAHP and the objective methods of the IE method have their own scope of adaptation. In most cases, the weight interval of the proposed hybrid method is the intersection of the weight intervals of the IAHP and IE methods. Thus, the proposed hybrid method is a good tradeoff between subjective and objective methods. The fuzzy character of the expert scoring is considered by the IAHP, and the fuzzy nature of the measured data is considered by the IE method.

The hybrid method combines the IAHP with IE method through the weight factor, which is a value from $[0,1]$. In this paper, the weight factor is 0.5 , but in practical application the choice of weight factor is not determined by an absolute optimal value. Considering the EUE in practice, the enumeration method is used to determine a relatively better value of the weight factor, or the best value of the factor will be optimized by the sensitivity analysis method according to the actual application.

\subsection{Comparison of different weight factors}

In order to determine the optimal weight factor, this section studies the impact of different weight factor values on the weight interval results. Figures 7 and 8 show lower bound and upper bound of weight intervals with respect to different weight factors ranging from 0 to 1 with the step size of 0.1 .

It can be observed from Figs. 7 and 8 that, when the weight factor is 0.5 in Figs. 7 and 8, the lower bound and upper bound of the weight for EUE is in the middle of the

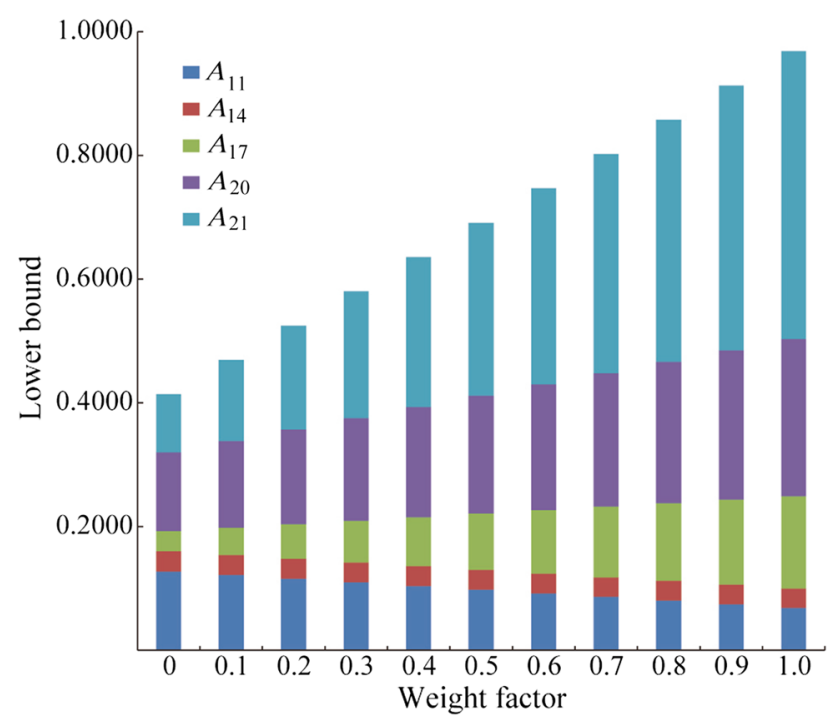

Fig. 7 Lower bound of weight for EUE with different weight factors

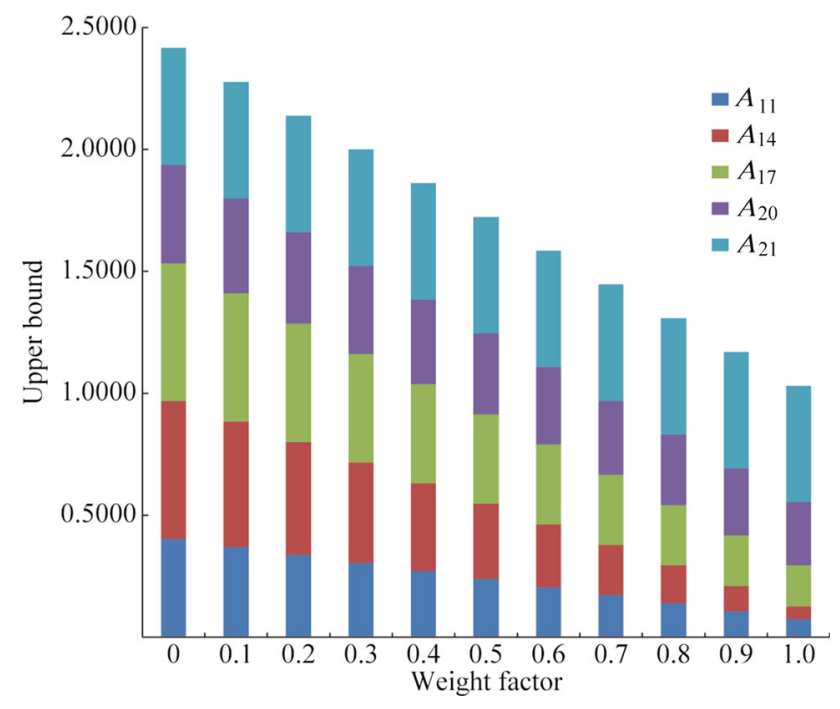

Fig. 8 Upper bound of weight for EUE with different weight factors

relative value. It should be a relatively balanced value, and is the optimal one.

Index $A_{21}$ shows the biggest change in Figs.7 and 8, which means that $A_{21}$ is the most sensitive index among energy efficiency indices.

Different factors derive different weights for EUE. The proposed hybrid method can cover the IAHP and IE methods by using different weight factors. When the factor is 1 , the hybrid method is reduced to the IAHP, and when the factor is 0 , the hybrid method is reduced to the IE method.

\section{Conclusion}

This paper proposes the EUE index system for energy efficiency, safety monitoring, and demand response, and explores the hybrid interval AHP-entropy method for optimizing the EUE index. The proposed EUE indices were described in detail and applied to an example industrial electricity user, showing the benefits and behaviors of the interval methods. The proposed hybrid interval AHP-entropy method can simultaneously consider the fuzziness of expert scoring and user data.

Acknowledgements This work was supported by the National Natural Science Foundation of China (No. NSFC 51361135704, No. 51377115) and the key project of the National Social Science Foundation (No. 12\&ZD208) and Tianjin Research Program of Application Foundation and Advanced Technology (No. 14JCYBJC21100).

Open Access This article is distributed under the terms of the Creative Commons Attribution 4.0 International License (http:// creativecommons.org/licenses/by/4.0/), which permits unrestricted use, distribution, and reproduction in any medium, provided you give 
appropriate credit to the original author(s) and the source, provide a link to the Creative Commons license, and indicate if changes were made.

\section{References}

[1] Jimin K, Taehoon H, Myeongsoo C et al (2015) An environmental and economic assessment for selecting the optimal ground heat exchanger by considering the entering water temperature. Energies 8:7752-7776

[2] Oyedepo SO, Fagbenle RO, Adefila SS et al (2014) Performance evaluation and economic analysis of a gas turbine power plant in Nigeria. Energy Conv Manag 79:431-440

[3] Ozan E (2014) Economic impacts of small-scale own generating and storage units, and electric vehicles under different demand response strategies for smart households. Appl Energy 126:142-150

[4] Smith R, Meng K, Dong ZY et al (2013) Demand response: a strategy to address residential air-conditioning peak load in Australia. J Mod Power Syst Clean Energy 1(3):223-230

[5] Armando MLS, Airton V, Claudio F et al (2014) Probabilistic evaluation of substation criticality based on static and dynamic system performances. IEEE Trans Power Syst 29(3):1410-1418

[6] Ma R, Li K, Li X et al (2015) An economic and low-carbon dayahead Pareto-optimal scheduling for wind farm integrated power systems. J Mod Power Syst Clean Energy 3(3):393-401

[7] Yan HG, Li B, Chen SS et al (2015) Future evolution of automated demand response system in smart grid for low-carbon economy. J Mod Power Syst Clean Energy 3(1):72-81

[8] Meng C, Xu D, Son Y et al (2014) An integrated simulation and AHP approach to vegetable grafting operation design. Comput Electron Agric 102:73-84

[9] Wang Y, Xi C, Zhang S et al (2014) A combination of extended fuzzy AHP and fuzzy GRA for government E-tendering in hybrid fuzzy environment. Sci World J. https://doi.org/10.1155/ 2014/123675

[10] Eugene RJ, Teresa W, Dan S (2014) A stochastic AHP decision making methodology for imprecise preferences. Inf Sci 270:192-203

[11] Wu J, Huang H, Cao Q (2013) Research on AHP with interval valued intuitionistic fuzzy sets and its application in multi criteria decision making problems. Appl Math Modeling 37(24):9898-9906

[12] Chen T, Jin Y, Qiu X et al (2014) A hybrid fuzzy evaluation method for safety assessment of food waste feed based on entropy and the analytic hierarchy process methods. Expert Sys Appl 41(16):7328-7337

[13] Etienne H, Eric D, Jean-Claude B (2014) Maximum entropy analysis of data simulation and practical aspects of time-resolved fluorescence measurements in the study of molecular interactions. J Mol Struct 1077:77-86

[14] Li CB, Xiao LW, Cao YJ et al (2014) Optimal allocation of multi-type FACTS devices in power systems based on power flow entropy. J Mod Syst Clean Energy 2(2):173-180

[15] Wang S, Han L, Zhang P (2014) Affine arithmetic-based DC power flow for automatic contingency selection with consideration of load and generation uncertainties. Electr Power Comput Syst 42:852-860

[16] Liu F, Zhang W, Zhang L (2014) A group decision making model based on a generalized ordered weighted geometric average operator with interval preference matrices. Fuzzy S Syst 246:1-18
[17] Byeong SA, Haechurl P (2014) Establishing dominance between strategies with interval judgments of state probabilities. Omega 49:53-59

[18] Gizem I, Erhan B, Tufan K (2013) The selection of technology forecasting method using a multi criteria interval valued intuitionistic fuzzy group decision making approach. Comput Ind Eng 65(2):277-285

[19] Agnieszka L, Artur G (2014) Model of unsteady heat exchange for intermittent heating taking into account hot water radiator capacity. Energy Build 76:176-184

[20] Deeper K, Samantaray SR (2014) Design of an advanced electric power distribution systems using seeker optimization algorithm. Int J Electr Power Energy Syst 63:196-217

[21] Saric AT, Stankvic AM (2006) An application of interval analysis and optimization to electric energy markets. IEEE Trans Power Syst 21(2):515-523

[22] Wu J, Luo Z, Zhang $\mathrm{N}$ et al (2015) A new uncertain analysis method and its application in vehicle dynamics. Mech Syst Signal Proc 50-51:659-675

[23] Hu Z, Bao Y, Chiong R et al (2015) Mid-term interval load forecasting using multi-output support vector regression with a memetic algorithm for feature selection. Energy 84:419-431

[24] Hao Q, Dipti S, Abbas K (2014) Uncertainty handling using neural network based prediction intervals for electrical load forecasting. Energy 73:916-925

[25] Tomoe E, Masahiro I (2015) Pairwise comparison based interval analysis for group decision aiding with multiple criteria. Fuzzy S Syst 274:79-96

[26] Hoang N (2015) A new knowledge-based measure for intuitionistic fuzzy sets and its application in multiple attribute group decision making. Expert Syst Appl 42(22):8766-8774

[27] Benedetto F, Giunta G, Mastroeni L (2015) A maximum entropy method to assess the predictability of financial and commodity prices. Digit Signal 46:19-31

Shouxiang WANG (SMIEEE'12) received the B.S. and M.S. degrees from Shandong University of Technology, Jinan, China, in 1995 and 1998, respectively, and Ph.D. degree from Tianjin University, Tianjin, China, in 2001, all in electrical engineering. He is currently a professor in the School of Electrical Information and Engineering at Tianjin University. His main research interests include distributed generation, microgrid and smart distribution system.

Leijiao GE received the Ph.D degree from Tianjin University, Tianjin, China, in 2016. He is currently an assisstant professor in the School of Electrical Information and Engineering at Tianjin University. His main research interests include smart distribution system, cloud computing and big data.

Shengxia CAI received the B.S. degree from Zhejiang University, Hangzhou, China, in 1993, and the M.Ec. and Ph.D. in economics from Nankai University, Tianjin, China, in 1999 and 2005, respectively. She is currently an associate professor in the Zhou Enlai School of Government at Nankai University. Her main research interests are energy management and energy policy.

Lei WU (SMIEEE'13) received the B.S. degree in electrical engineering and the M.S. degree in systems engineering from Xi'an Jiaotong University, Xi'an, China, in 2001 and 2004, respectively, and the $\mathrm{Ph} . \mathrm{D}$. degree in electrical engineering from Illinois Institute of Technology, Chicago, IL, USA, in 2008. Presently, he is an associate professor in the Electrical and Computer Engineering Department at Clarkson University, Potsdam, NY, USA. His research interests include power systems optimization and economics. 